\title{
Use of Time- and Frequency-Domain Approaches for Damage Detection in Civil Engineering Structures
}

\author{
V. H. Nguyen, ${ }^{1}$ J. Mahowald, ${ }^{1}$ S. Maas, ${ }^{1}$ and J.-C. Golinval ${ }^{2}$ \\ ${ }^{1}$ Faculty of Science, Technology and Communication, University of Luxembourg, 6 rue Richard Coudenhove-Kalergi, \\ 1359 Luxembourg, Luxembourg \\ ${ }^{2}$ Department of Aerospace and Mechanical Engineering, University of Liege, 1 Chemin des Chevreuils, B52, 4000 Liège, Belgium
}

Correspondence should be addressed to V. H. Nguyen; vietha.nguyen@uni.lu

Received 27 July 2013; Accepted 12 December 2013; Published 15 June 2014

Academic Editor: Nuno Maia

Copyright (C) 2014 V. H. Nguyen et al. This is an open access article distributed under the Creative Commons Attribution License, which permits unrestricted use, distribution, and reproduction in any medium, provided the original work is properly cited.

\begin{abstract}
The aim of this paper is to apply both time- and frequency-domain-based approaches on real-life civil engineering structures and to assess their capability for damage detection. The methodology is based on Principal Component Analysis of the Hankel matrix built from output-only measurements and of Frequency Response Functions. Damage detection is performed using the concept of subspace angles between a current (possibly damaged state) and a reference (undamaged) state. The first structure is the Champangshiehl Bridge located in Luxembourg. Several damage levels were intentionally created by cutting a growing number of prestressed tendons and vibration data were acquired by the University of Luxembourg for each damaged state. The second example consists in reinforced and prestressed concrete panels. Successive damages were introduced in the panels by loading heavy weights and by cutting steel wires. The illustrations show different consequences in damage identification by the considered techniques.
\end{abstract}

\section{Introduction}

Modal identification and damage detection methods using output-only measurements are very attractive in the field of structural health monitoring (SHM) when the ambient excitation is unknown (e.g., in civil engineering structures submitted to wind or traffic excitation). A review of vibrationbased health monitoring methods can be found in $[1,2]$.

For the purpose of modal analysis, time-domain methods such as the stochastic subspace identification (SSI) method are currently applied. For damage detection, methods such as Principal Component Analysis (PCA) and Second-Order Blind Identification (SOBI) were also recently developed. The robustness of these methods was improved by making use of the Hankel matrix instead of the observation matrix leading to the following variant approaches: Enhanced PCA, Null Subspace Analysis (NSA), or Enhanced SOBI. Their efficiency has been demonstrated in earlier studies mainly on numerical examples and laboratory experiments $[3,4]$. They were also tested successfully on industrial examples to perform machine condition monitoring using a reduced set of sensors [5].
The aim of this paper is to present some applications of a PCA-based damage detection technique to civil engineering structures. The first structure consists in the Champangshiehl Bridge which is a two-span concrete box girder bridge located in Luxembourg. Next, precast reinforced and prestressed concrete slabs are considered. A sensitivity analysis for PCA in the frequency domain is used for the purpose of damage localization.

\section{Dynamic Feature Extraction Using Principal Component Analysis}

Let us consider a dynamical system characterized by a set of vibration measurements collected in the observation matrix $\mathrm{X}$ :

$$
\mathbf{X}=\left[\begin{array}{llllll}
\mathbf{x}_{1} & \mathbf{x}_{2} & \cdots & \mathbf{x}_{k} & \cdots & \mathbf{x}_{N}
\end{array}\right], \quad \mathbf{x}_{k} \in \mathbf{R}^{m},
$$

where $\mathbf{x}_{k}$ is the output vector at time step $k, m$ is the number of output sensors, and $N$ is the number of time samples. Principal Component Analysis (PCA) aims to reduce the dimensionality of the observed data while preserving most of 
information contained in the data set [6]. This is realized by finding $p$ principal axes, which allow a data projection onto the $p$-dimensional subspace so that the mean square distance between the original points and corresponding projection is minimal. The dimension $p$ corresponding to the number of principal components defines the rank of matrix $\mathbf{X}$ and is directly related to the order of the system. In practice, PCA is often performed by singular value decomposition (SVD) of matrix; that is,

$$
\mathbf{X}=\mathbf{U} \boldsymbol{\Sigma} \mathbf{V}^{\mathrm{T}}
$$

where $\mathbf{U}$ and $\mathbf{V}$ are orthonormal matrices, the columns of $\mathbf{U}$ defining the principal components (PCs). The order $p$ of the system is determined by selecting the first $p$ nonzero singular values in $\Sigma$ which have a significant magnitude ("energy") as described in [7].

The null subspace (NSA) and enhanced-PCA method (EPCA) proposed in $[3,4]$, respectively, are variant methods of the PCA method obtained by exploiting Hankel matrices of the dynamical system [8]. The data-driven block Hankel matrix is defined in (3), where $2 i$ is a user-defined number of row blocks, each block contains $m$ rows (number of measurement sensors), and $j$ is the number of columns (practically $j=N-2 i+1$ ). The Hankel matrix $\mathbf{H}_{1,2 i}$ consists of $2 \mathrm{im}$ rows and is split into two equal parts of $i$ block rows, which represent past and future data, respectively. Compared to the observation matrix $\mathbf{X}$, the Hankel matrix is built using time-lagged vibration signals and not instantaneous representations of responses. This enables taking into account time correlations between measurements when current data depend on past data. Therefore, the objective pursued here in using block Hankel matrices rather than observation matrices is to improve the sensitivity of the detection method:

$$
\mathbf{H}_{1,2 i}=\left[\begin{array}{ccccc}
\mathbf{x}_{1} & \mathbf{x}_{2} & \cdots & \cdots & \mathbf{x}_{j} \\
\mathbf{x}_{2} & \mathbf{x}_{3} & \cdots & \cdots & \mathbf{x}_{j+1} \\
\cdots & \cdots & \cdots & \cdots & \cdots \\
\mathbf{x}_{i} & \mathbf{x}_{i+1} & \cdots & \cdots & \mathbf{x}_{i+j-1} \\
\hline \mathbf{x}_{i+1} & \mathbf{x}_{i+2} & \cdots & \cdots & \mathbf{x}_{i+j} \\
\mathbf{x}_{i+2} & \mathbf{x}_{i+3} & \cdots & \cdots & \mathbf{x}_{i+j+1} \\
\cdots & \cdots & \cdots & \cdots & \cdots \\
\mathbf{x}_{2 i} & \mathbf{x}_{2 i+1} & \cdots & \cdots & \mathbf{x}_{2 i+j-1}
\end{array}\right] \equiv\left[\frac{\mathbf{H}_{p}}{\mathbf{H}_{f}}\right] \equiv \frac{\text { "past" }}{\text { "future" }}
$$

where the subscripts of $\mathbf{H}_{1,2 i}$ denote the subscript of the first and last element of the first column in the block Hankel matrix.

\section{Damage Detection Based on the Concept of Subspace Angle}

The principal components contained in matrix $\mathbf{U}$ span a subspace, which characterizes the dynamic state of the system. Without any damage or variation of environmental conditions, the characteristic subspace $\mathbf{U}$ remains unchanged. Any change in the dynamic behaviour caused by a modification of the system state modifies consequently its characteristic subspace. This change may be estimated using the definition

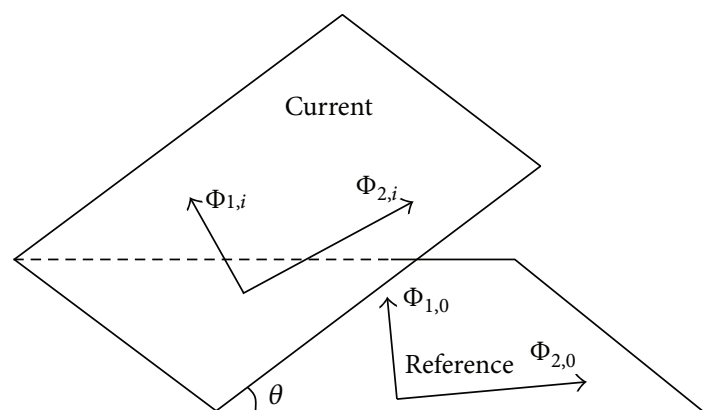

Figure 1: Angle $\theta$ formed by active subspaces according to the reference and current states, due to a dynamic change.

of subspace angles [9]. As illustrated by a two-dimensional case in Figure 1, the concept of subspace angle can be seen as a tool to quantify existing spatial coherence between two data sets resulting from observations of a vibration system. In the figure, an active subspace is built from two principal components (column vectors) of matrix $\mathbf{U}$.

\section{Damage Detection in the Champangshiehl Bridge}

4.1. Description of the Bridge. The Champangshiehl Bridge shown in Figure 2 is a two-span concrete box girder bridge built in 1966 and located in the centre of Luxembourg. The bridge has a total length of $102 \mathrm{~m}$ divided into two spans of $37 \mathrm{~m}$ and $65 \mathrm{~m}$, respectively. It is prestressed by 112 steel wires as illustrated in Figure 2(b). Before its complete destruction, the bridge was monitored and a series of damages were artificially introduced as summarized in Table 1 . The four damage cases considered are illustrated in Figures 3(a)-3(d).

The measurement setup considered in the present work is given in Figure 4. Ten sensors were located on each side $A$ and $B$ of the deck (the distance between each sensor is about $10 \mathrm{~m}$ ). Vibration monitoring under impact excitation was performed on the healthy structure and at each damage state. More detailed descriptions of the bridge can be found in $[10]$.

4.2. Analysis Results. The bridge may be analyzed through a well established modal identification method proposed in [11] which relies on the use of stochastic subspace identification (SSI). Two first eigenfrequencies obtained for the four damage cases (D1-D4) are compared to the eigenfrequencies of the healthy structure as reported in Table 2.

Table 2 shows that the decrease of the eigenfrequencies is proportional to the damage level for damage cases D1, D3, and D4. Only damage case D2 exhibits a different behaviour as the first eigenfrequency increases by an amount of $1.6 \%$ with respect to the healthy case. Moreover, the second eigenfrequency is affected by the larger decrease (5.42\%) of all the damage states. This is in good agreement with an earlier analysis reported in [12].

The application of the concept of subspace angle on the Champangshiehl Bridge data allows detecting all the damage 


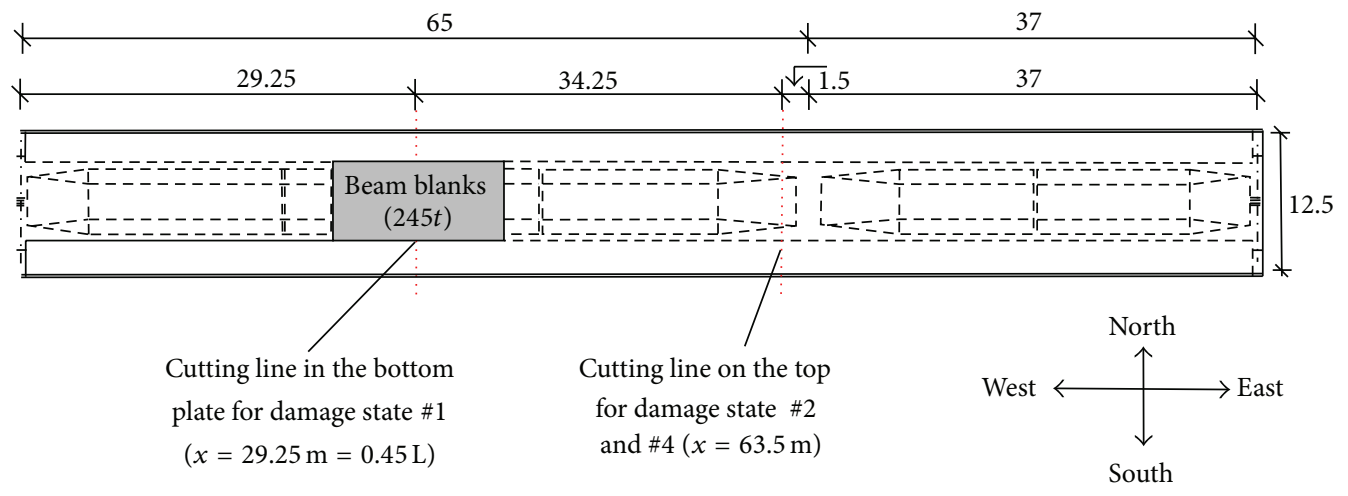

(a) Longitudinal section of the bridge

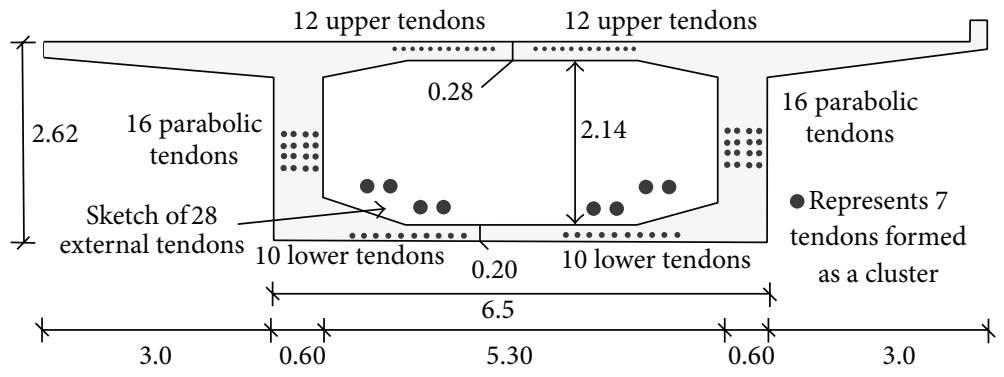

- Represents 7 tendons formed as a cluster

(b) Schematic cross section of the box girder with location of the tendons

Figure 2: The Champangshiehl Bridge.

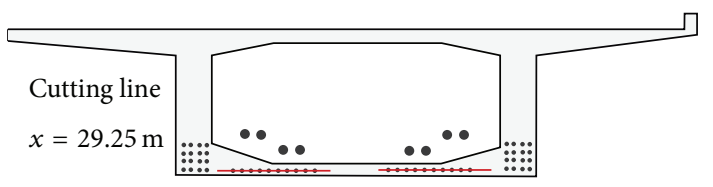

(a) Damage case \#1

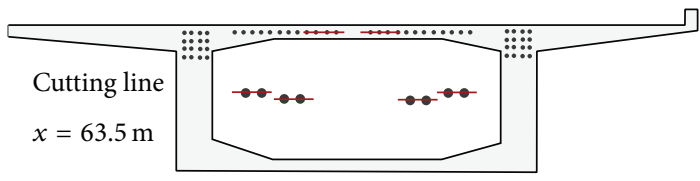

(c) Damage case \#3

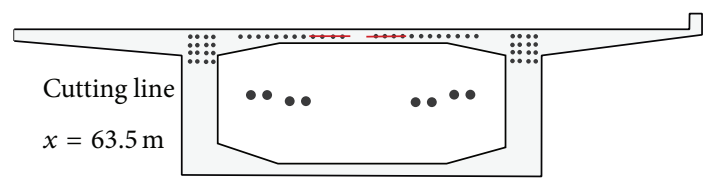

(b) Damage case \#2

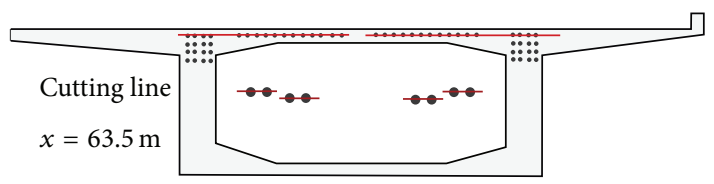

(d) Damage case \#4

Figure 3: Damage scenarios.

TABLE 1: Description of the damage scenarios according to the cutting sections shown in Figure 2.

\begin{tabular}{|c|c|c|c|}
\hline \multirow{2}{*}{ State } & \multirow{2}{*}{ Damage } & \multicolumn{2}{|c|}{ Percentage cutting ( $100 \%$ equals all tendons in the defined section cut) } \\
\hline & & $0.45 \mathrm{~L}$ & Over the pylon \\
\hline$\# 0$ & Undamaged state & & \\
\hline$\# 1$ & $\begin{array}{l}\text { Cutting straight lined tendons in the lower part, at } \\
0.45 \mathrm{~L} \text { ( } 20 \text { tendons) }\end{array}$ & $33.7 \%$ & $0 \%$ \\
\hline \#2 & $\begin{array}{l}\# 1+\text { cutting } 8 \text { straight lined tendons in the upper part, } \\
\text { over the pylon }\end{array}$ & $33.7 \%$ & $12.6 \%$ \\
\hline$\# 3$ & $\# 2+$ cutting external tendons (56 wires) & $46.1 \%$ & $24.2 \%$ \\
\hline \#4 & $\begin{array}{l}\# 3+\text { cutting } 16 \text { straight lined tendons in the upper part } \\
\text { and } 8 \text { parabolic tendons }\end{array}$ & $46.1 \%$ & $62.12 \%$ \\
\hline
\end{tabular}


TABLE 2: Change in the eigenfrequencies (identified by SSI).

\begin{tabular}{lcccc}
\hline & & $f_{1}$ & & $f_{2}$ \\
& Value $(\mathrm{Hz})$ & $\Delta f_{1}(\%)$ & Value $(\mathrm{Hz})$ & $\mathbf{5 . 5 4}$ \\
Healthy & $\mathbf{1 . 9 2}$ & & 5.45 & -1.62 \\
D1 & 1.87 & -2.6 & 5.24 & -5.42 \\
D2 & 1.95 & 1.6 & 5.39 & -2.71 \\
D3 & 1.82 & -5.21 & 5.3 & -4.33 \\
D4 & 1.75 & -8.85 & & \\
\hline
\end{tabular}

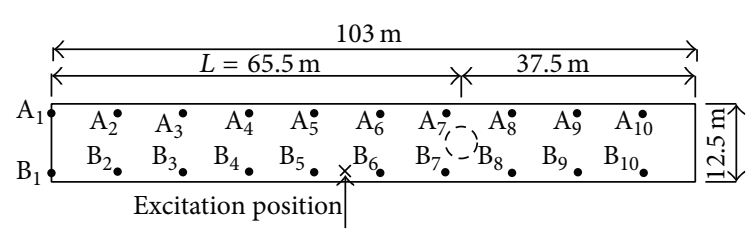

FIGURE 4: Location of the sensors on the bridge deck.

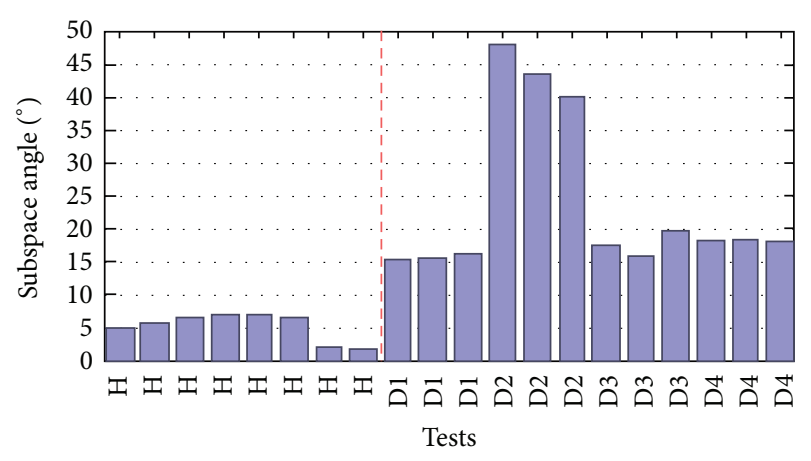

FIGURE 5: Damage detection results using EPCA.

cases (D1-D4) using the single first principal component (PC) of the Hankel matrix. The detection remains good and even more evident when 2, 3, and 4 PCs are used.

On the other hand, the use of more PCs (higher than 4) deteriorates the quality of the distinction between the damaged and the healthy states. Indeed, the highest PCs (associated with small singular values, that is, low energy) come from noise present in the data and are not dynamic features of the system. As an example, the detection results obtained on the basis of 3 PCs are shown in Figure 5. In this figure, a total of 20 tests were considered: eight tests on the healthy structure $(\mathrm{H})$ and twelve tests corresponding to the four levels of damages D1-D4. It can be observed that all the damage cases are well detected and that damage cases D2 present the largest damage indexes.

\section{Damage Detection on Precast Panels}

5.1. Description of the Panels. The two investigated panels are manufactured by the Luxembourg company ECHOLUX and both are of the same type (one prestressed concrete $(\operatorname{PrC})$, one special fabricated nonprestressed, reinforced concrete (RC) for testing purposes only). They are made of concrete C50/60 with an elastic modulus of $42700 \mathrm{~N} / \mathrm{mm}^{2}$ and a measured compressive strength of $58.3 \mathrm{~N} / \mathrm{mm}^{2}$ (quality control of manufacturer). The quality of the reinforcement is St 1470/1670 and the corresponding elastic modulus $205000 \mathrm{~N} / \mathrm{mm}^{2}$. In the upper section of the panel, there are 4 wires with a diameter of $5 \mathrm{~mm}$ and in the lower Section 12 wires with a diameter of $7 \mathrm{~mm}$. Before testing, the concrete at the bottom side in the middle of the slab along axis $C$ (Figure 6(b)) was removed, as shown in Figure 6(a), to give access to the reinforcement for the later procedures of cutting tendons.

Both static and dynamic tests were performed on the slabs to compare their behavior in each condition [13]. The dynamic responses were measured using impact testing. The sample rate of the data acquisition is set to $200 \mathrm{~Hz}$; signals were recorded during 8 seconds after the introduction of impact. The measurements are set with a quite dense grid ( $\Delta=14.55 \mathrm{~cm}$, Figure 7$)$ for the sake of studying damage localization later. There are 45 impact points at each side of the slabs and three accelerometers (Ref. 1-Ref. 3 in Figure 7) are used to capture dynamic responses. So, in each condition, we have 3 sets of data containing 90 signals.

Damages were introduced by static mass loading (Figure 6(b)), cutting of steel wires and are resumed in Table 3.

5.2. Analysis of the Results. Relating to frequency, damages show influence principally on the first component. Table 5 presents the first eigenfrequency shift, identified by the peak picking and SSI methods, respectively.

The results obtained in Table 5 for the RC slab show a good agreement between the peak picking and SSI methods. It shows a clear decrease of frequency values following the increasing levels of damage. However, for the PrC slab, the eigenfrequencies vary very slightly between different conditions; only the intact state $(\# 0)$ and the state before the failure $\left(\# 3^{*}\right)$ are clearly distinct. The values identified by SSI cannot classify levels $\# 0$ to $\# 2^{*}$. This is consistent with the observations and cracking described in Table 4: no change is noticed between state $\# 0$ to $\# 1^{*}$. It reveals that, in comparison with the RC slab, apparent damage occurs very late in the PrC slab; the crack formation and hence the deformation are negligible until failure, which makes the detection more difficult.

Before the implementation of the static and dynamic tests, cracking loads were calculated for each structure. For the RC slab, the cracking load is expected for a load of two steel weights $\left(G_{1}\right.$ and $G_{2}$ in Figure 6(b)) without cutting of 


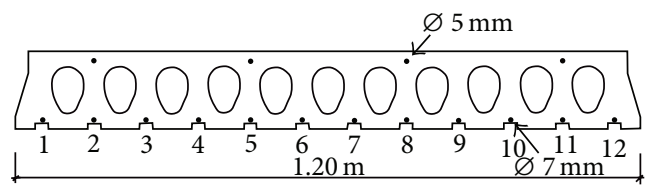

- Steel reinforcement

(a) Cross section of the panels

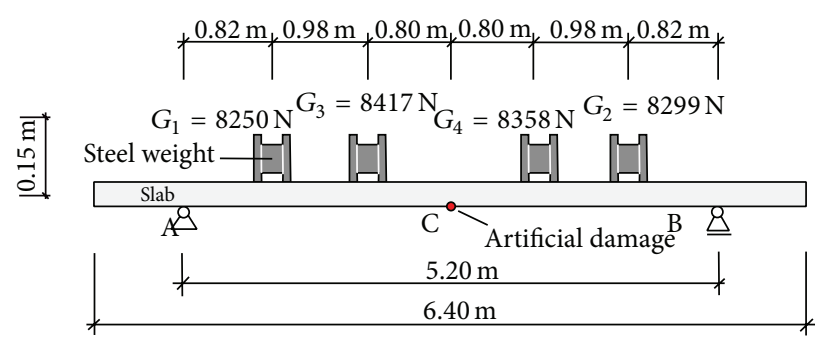

(b) Schema of loading and location of cracks (point C)

Figure 6: Panel structure and experiment schema.

TABLE 3: Damage scenarios.

\begin{tabular}{|c|c|c|c|}
\hline Number & Damage scenario & Cutting percentage & Remark \\
\hline$\# 0$ & Intact state-no damage & - & \multirow{5}{*}{$\begin{array}{l}\text { Later we consider states } \\
\# 0, \# 0^{*}, \# 1^{*}, \# 2^{*}, \# 3^{*} . \\
{ }^{*} \text { denotes a state after } \\
\text { loading and then } \\
\text { removing of } 4 \text { heavy } \\
\text { weights from the slab } \\
\text { (shown in Figure } 6(\mathrm{~b}) \text { ) }\end{array}$} \\
\hline$\# 1$ & $\begin{array}{l}\text { Cutting of } 2 \text { tendons (numbers } 6 \text { and } 7 \text {-refer to } \\
\text { Figure } 6(a) \text { ) }\end{array}$ & $16.7 \%$ & \\
\hline \#2 & Cutting of 4 tendons (numbers $6,7,2$, and 11) & $33.3 \%$ & \\
\hline$\# 3$ & $\begin{array}{l}\text { Cutting of } 6 \text { tendons (numbers } 6,7,2,11,4 \text {, and } \\
9 \text { ) }\end{array}$ & $50 \%$ & \\
\hline$\# 4$ & $\begin{array}{l}\text { Cutting of } 8 \text { tendons (numbers } 6,7,2,11,4,9,3 \text {, } \\
\text { and 10) }\end{array}$ & $66.7 \%$ & \\
\hline
\end{tabular}

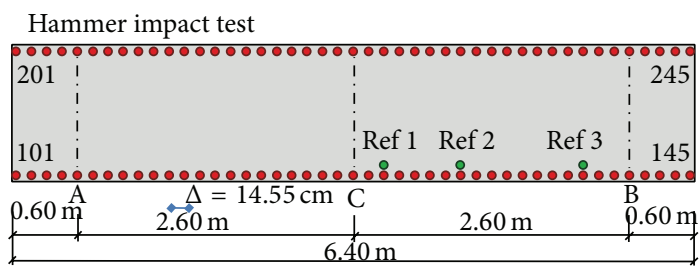

- Excitation

- Measurement

Figure 7: Measurement setup: impact point (101-145 and 201-245) and accelerometer positions (Ref. 1-Ref. 3).

any wires. Contrarily, the cracking load for the $\operatorname{PrC}$ slab is expected for an additional load of four steel weights $\left(G_{1}, G_{2}\right.$, $G_{3}$, and $G_{4}$ ) and cutting of 6 to 7 wires.

As presented in Figure 8, EPCA detects dynamic change in the RC slab from the loading of 2 masses, what corresponds already to the cracking load, while visible cracks are noticed only after the loading of 4 masses. Furthermore, the results distinguish clearly the tests before and after cutting tendons: larger subspace angles are obtained for the last cases. All signals processed here were measured after a procedure of charging then removing masses. Each condition is represented by 3 sets of measurement; one set of measurement in the intact state is provided for reference data.

For the PrC slab, it is theoretically proven that the cracking load can be reached much later with respect to the $\mathrm{RC}$ slab. Only a hairline crack occurs after the loading of 4 masses in addition to the cutting of 4 tendons $\left(\# 2^{*}\right)$. In this circumstance, for a more precise comparison between

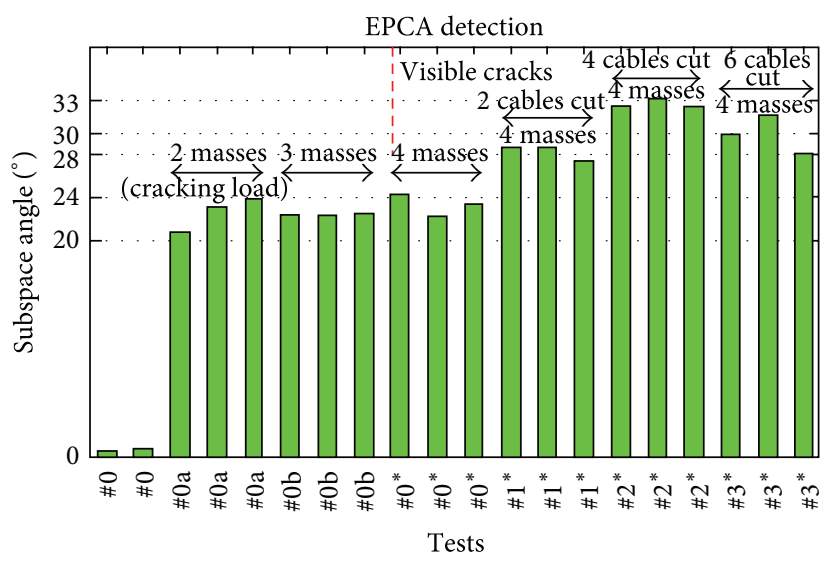

FIGURE 8: EPCA detection for the RC slab (always unloaded state).

different conditions in the PrC slab, we examine only the correlation of states after a procedure of loading then removing the 4 masses. All data refer to the intact state $\# 0^{*}$ after removing the masses. As presented in Figure 9, the EPCA method is able to detect well the damages caused in the slab. As in the visible observations, subspace angles do not reveal much difference between damages $\# 1^{*}$ to $\# 3^{*}$.

5.3. Localization of Damage. In this paper, damage localization in beam-like structures is based on the use of sensitivity analysis of measurements. A review on modal updating methods including the sensitivity of both frequencies and mode-shapes is given in [14]. Natural frequency sensitivity has been used extensively for the purpose of damage localization. However, most of the methods based 
TABLE 4: Description of damages.

\begin{tabular}{lll}
\hline Number & Reinforced concrete (RC) slab & Prestressed concrete (PrC) slab \\
\hline$\# 0$ & No damage & No damage \\
$\# 0^{*}$ & Appearance of a decisive crack pattern, large creep & No crack observed \\
$\# 1^{*}$ & No further cracks, current cracks grow and also creep & No crack observed, no considerable deformation \\
$\# 2^{*}$ & As above & Appearance of a hairline crack, minimal deformation \\
$\# 3^{*}$ & As above & As above \\
$\# 4^{*}$ & Collapse & Collapse \\
\hline
\end{tabular}

TABLE 5: The shift of the first eigenfrequency $(\mathrm{Hz})$ from the intact state until before the collapse (always unloaded state).

\begin{tabular}{|c|c|c|c|c|c|c|c|c|c|c|}
\hline \multirow[b]{2}{*}{ State } & \multicolumn{5}{|c|}{ RC slab } & \multicolumn{5}{|c|}{ PrC slab } \\
\hline & $\# 0$ & $\# 0^{*}$ & $\# 1^{*}$ & $\# 2^{*}$ & $\# 3^{*}$ & $\# 0$ & $\# 0^{*}$ & $\# 1^{*}$ & $\# 2^{*}$ & $\# 3^{*}$ \\
\hline$f$ by peak picking & 11 & 9.18 & 8.07 & 7.85 & 7.69 & 11.75 & 11.70 & 11.65 & 11.65 & 11.55 \\
\hline$f$ by SSI & 11 & 9.20 & 8.00 & 7.70 & 7.60 & 11.73 & 11.65 & 11.61 & 11.56 & 11.33 \\
\hline
\end{tabular}

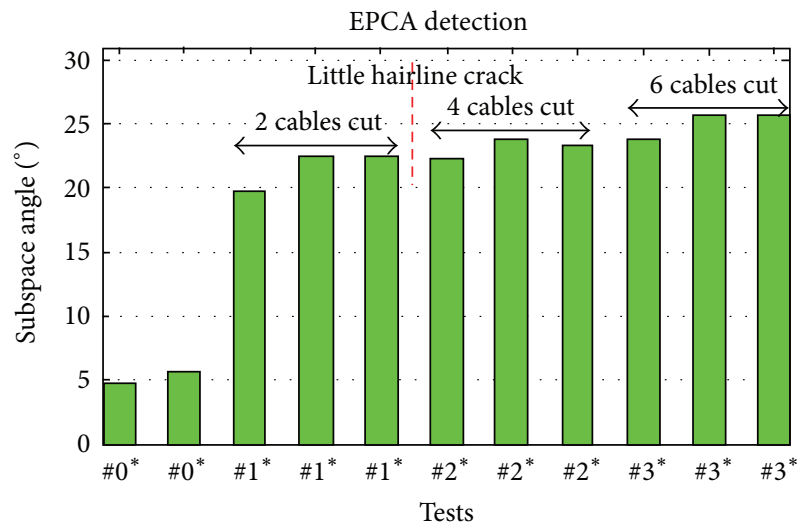

Figure 9: EPCA detection for the PrC slab (always unloaded state).

on frequency sensitivity with respect to damage variables require an accurate analytical model. In [15], an extension of the frequency sensitivity approach is proposed to eliminate this requirement. However, an optimization procedure is still needed to estimate the unknown system matrices through an identified model using input-output measurement data.

Natural frequencies are known to be very efficient in characterizing changes in dynamical systems. Mode-shapes are considered efficient to recognize spatial changes, since they condense most of the deformation database of the structure. In the present work, the sensitivity of mode-shapes is considered. However, the construction of an analytical model is not necessary for the localization procedure.

5.3.1. Index for Localization. In the previous sections, the SSI and EPCA methods were used in the time-domain for modal identification and damage detection. Damage may be located based on the estimation of flexibility from the identified mode-shapes as presented in [10]. In this section, Principal Component Analysis (PCA) is used for damage localization based on a sensitivity analysis in the frequency-domain. The technique is described in earlier works $[4,16,17]$ and is summarized here briefly.
Let us consider the Frequency Response Functions (FRFs) $\mathbf{H}^{s}(\omega)$ for a single input at location $s$ :

$$
\mathbf{H}^{s}(\omega)=\left[\begin{array}{llll}
\mathbf{h}\left(\omega_{1}\right) & \mathbf{h}\left(\omega_{2}\right) & \cdots & \mathbf{h}\left(\omega_{N}\right)
\end{array}\right],
$$

where vector $\mathbf{h}\left(\omega_{k}\right)$ is of dimension $m$ (the number of measured coordinates) and $N$ is the number of frequency lines. The rows of $\mathbf{H}^{s}$ represent the responses at the measured degrees of freedom (DOFs), while the columns are "snapshots" of the FRFs at different frequencies. We will assume that the dynamical system matrices depend on a vector of parameters $\mathbf{p}$. This vector of parameters may consist of system parameters or state variables. We can assess its principal components through Singular Value Decomposition (SVD) as represented in (2). As $\mathbf{H}^{s}$ belongs to the frequency-domain, the left singular vectors in $\mathbf{U}$ give spatial information, the right singular vectors in $\mathbf{V}$ represent modulation functions depending on frequency, and the diagonal matrix of singular values $\boldsymbol{\Sigma}$ contains scaling parameters of descending order $\sigma_{1}>\sigma_{2}>\cdots>\sigma_{m}$. In other words, the SVD of $\mathbf{H}^{s}$ separates information depending on space and frequency.

From (2), a sensitivity analysis can be performed by taking the derivative of the observation matrix $\mathbf{H}^{s}$ with respect to $\mathbf{p}$ :

$$
\frac{\partial \mathbf{H}^{s}}{\partial \mathbf{p}}=\frac{\partial \mathbf{U}}{\partial \mathbf{p}} \boldsymbol{\Sigma} \mathbf{V}^{\mathrm{T}}+\mathbf{U} \frac{\partial \boldsymbol{\Sigma}}{\partial \mathbf{p}} \mathbf{V}^{\mathrm{T}}+\mathbf{U} \boldsymbol{\Sigma} \frac{\partial \mathbf{V}^{\mathrm{T}}}{\partial \mathbf{p}} .
$$

Through this equation, the sensitivity of the system dynamic response shows its dependence on the sensitivity of each SVD term. Junkins and Kim [18] developed a method to compute the partial derivatives of SVD factors. Here, for the sake of localization, we are more particularly interested in spatial information contained in the left singular vector $\mathbf{U}$; its sensitivity with respect to a parameter $p_{k}$ is simply given by the following equation:

$$
\begin{gathered}
\frac{\partial \mathbf{U}_{i}}{\partial p_{k}}=\sum_{j=1}^{m} \alpha_{j i}^{k} \mathbf{U}_{j} \text { with } \\
\alpha_{j i}^{k}=\frac{1}{\sigma_{i}^{2}-\sigma_{j}^{2}}\left[\sigma_{i}\left(\mathbf{U}_{j}^{\mathrm{T}} \frac{\partial \mathbf{H}^{s}}{\partial p_{k}} \mathbf{V}_{i}\right)+\sigma_{j}\left(\mathbf{U}_{i}^{\mathrm{T}} \frac{\partial \mathbf{H}^{s}}{\partial p_{k}} \mathbf{V}_{j}\right)^{\mathrm{T}}\right]
\end{gathered}
$$



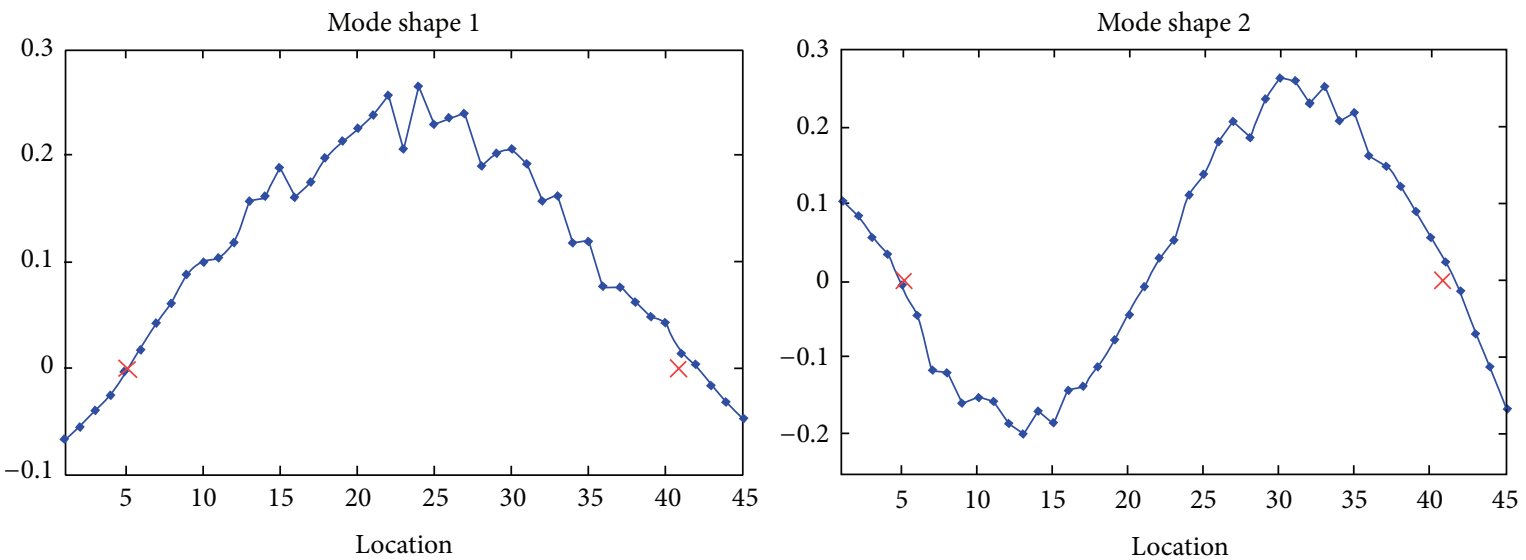

(a) Mode-shapes obtained by SSI
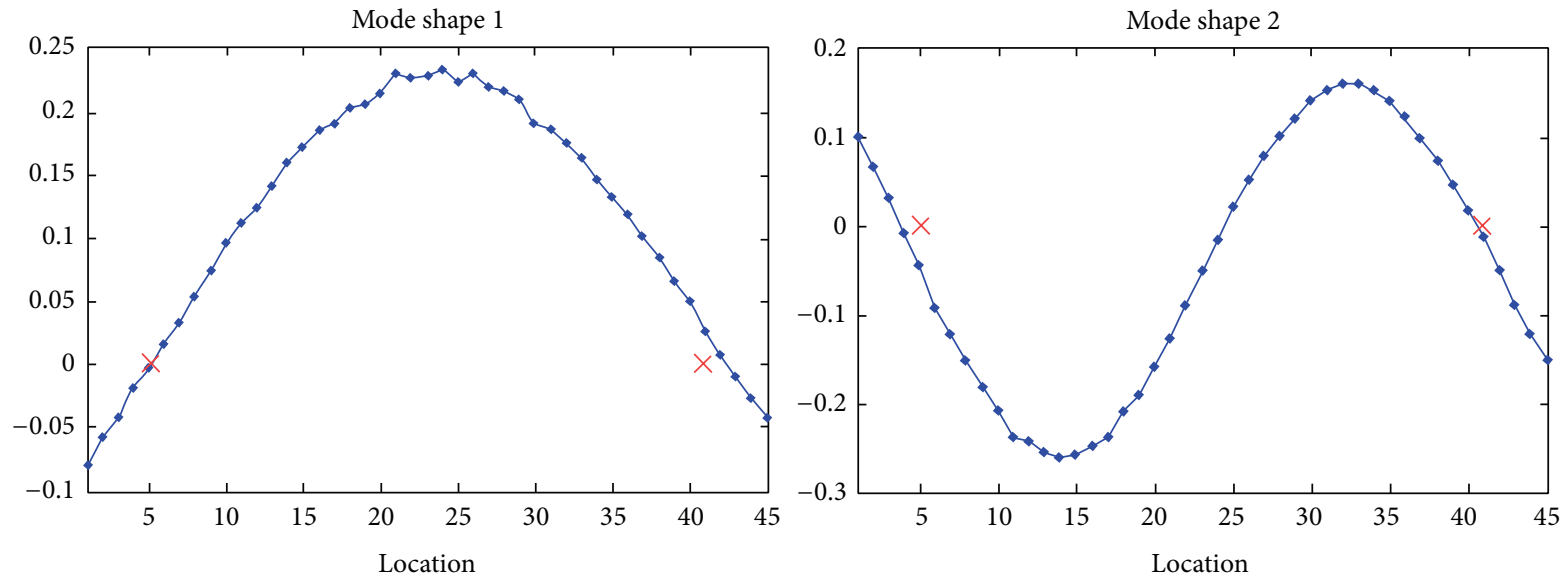

(b) Mode-shapes obtained by the sensitivity analysis

FIGURE 10: Comparison of mode-shapes obtained by SSI and the sensitivity analysis ( $\times$ : position of support).

It is shown in [18] that the diagonal coefficients $\alpha_{i i}^{k}$ keep only their imaginary part (their real parts are empty).

So, the sensitivity of the $i$ th principal component can be computed through coefficients $\alpha_{j i}^{k}$ which depend on an unknown $\partial \mathbf{H}^{s} / \partial p_{k}$. It is proven in [16] that when the system matrices are symmetric, if parameter of interest is some coefficient $k_{e}$ of the stiffness matrix, the sensitivity of the FRF matrix may be simply determined by the following formula:

$$
\frac{\partial \mathbf{H}^{s}}{\partial p_{k}}=-\mathbf{H}_{k_{e}} \cdot \mathbf{H}_{k_{e}, s}
$$

where $\mathbf{H}_{k_{e}}$ is just the row vector corresponding to coefficient $k_{e}$ in the FRF matrix in (4) and $H_{k_{e}, s}$ is the $s$ element of this vector.

Once $\partial \mathbf{H}^{s} / \partial p_{k}$ has been computed, the sensitivity of the left singular vectors is a good candidate for resolving localization problems of linear-form structures, for example, chain-like or beam-like structures. In each working condition of the system, we can compute the sensitivity $\partial \mathbf{U}_{i} / \partial p_{k}$. The reference state is denoted by $\partial \mathbf{U}_{i}^{R} / \partial p_{k}$, and the deviation of the current condition may be assessed as follows:

$$
\Delta \frac{\partial \mathbf{U}_{i}}{\partial p_{k}}=\frac{\partial \mathbf{U}_{i}}{\partial p_{k}}-\frac{\partial \mathbf{U}_{i}^{R}}{\partial p_{k}} .
$$

The last vector allows the maximization of useful information for damage localization.

5.3.2. Application on the Precast Panels. First, let us note that the sensitivity analysis of the FRF data allows extracting structural mode-shapes thanks to the principal component vectors contained in matrix $\mathbf{U}$. For the sake of conciseness, only the signals coming from one slab side are used here (from points 101 to 145 in Figure 7). Figure 10 compares the mode-shapes identified through SSI and the sensitivity analysis, respectively. It clearly shows that the mode-shapes obtained by the sensitivity analysis are smoother than by SSI. The SSI modes show larger variations at points of high amplitude.

As stated before, damage produces a crack pattern in the middle of the slab. So it is expected that the damage 


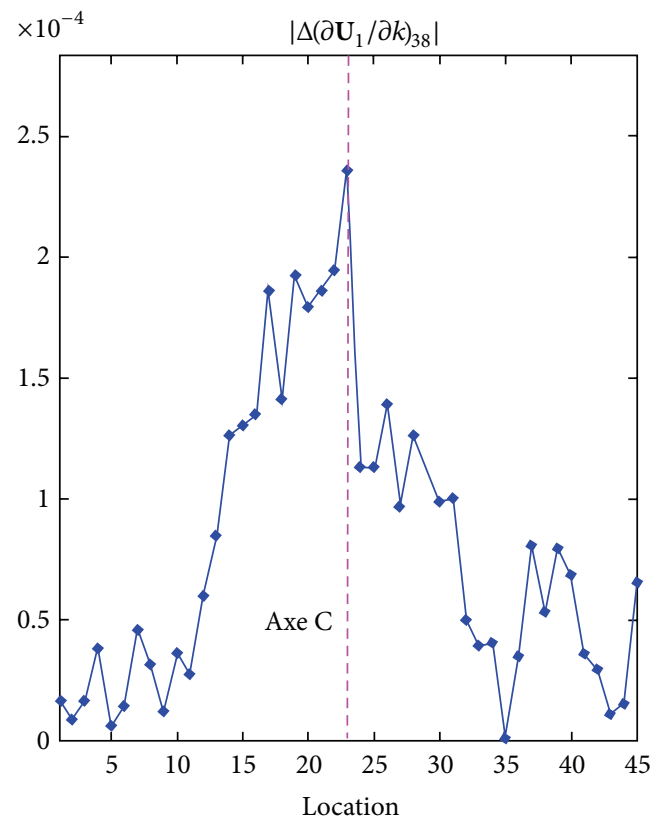

(a) Based on mode 1

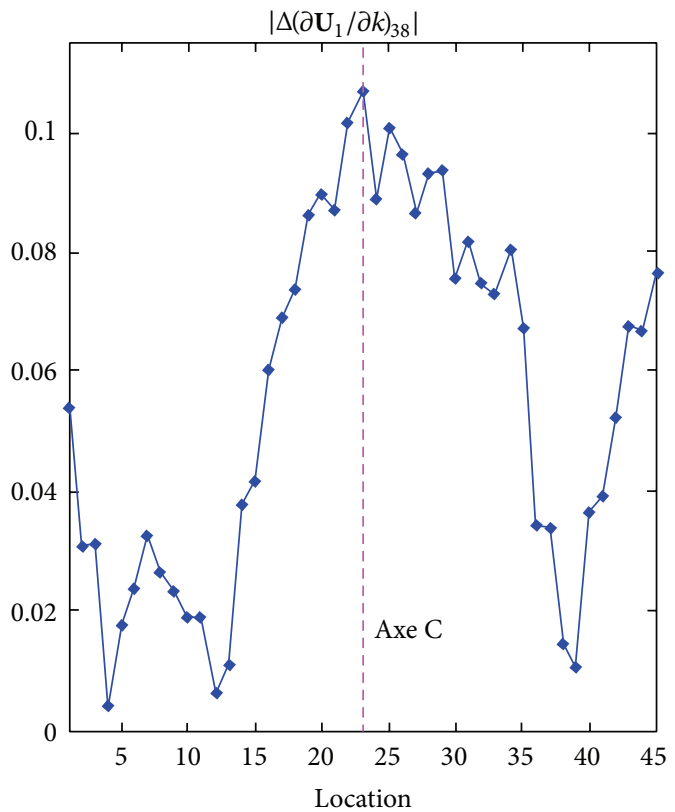

(b) Based on mode 2

FIgure 11: Damage localization in the RC slab for damage \#2*

localization procedure will point out damage around this zone, that is, along axis $\mathrm{C}$ passing through point 23 (see Figure 7) which marks the middle of the slab.

Let us remind you that in PCA, a large number of data are one of the requirements so that a principal component in $\mathrm{U}$ converges to a modal vector; so a frequency range should be chosen large enough for a sufficient observation of data in $\mathbf{H}^{s}(\omega)$. For the RC slab, the frequency range of $[4 \mathrm{~Hz}-26 \mathrm{~Hz}]$ corresponding to mode 1 is first selected to eliminate lowfrequency noise and higher frequency modes.

The results for $\left|\Delta\left(\partial \mathbf{U}_{1} / \partial p_{k}\right)\right|$ shown in Figure 11 are obtained from the set of measurement number 3 . As the sensor was located at point 38 for this set of measurement, parameter $p_{k}$ is chosen to correspond to $k_{38}$ according to the 38th element of the "experimental" stiffness matrix. The "undamaged" vector of $\partial \mathbf{U}_{1} / \partial p_{k}$ is extracted from state \#0 which is considered as reference. The diagrams of $\left|\Delta\left(\partial \mathbf{U}_{1} / \partial p_{k}\right)\right|$ in Figure 11 show for both modes 1 and 2 that the highest peaks are located close to point 23 (axe C) where the cracks gather. To take into account higher frequency component (mode 2), the frequency range of $[4 \mathrm{~Hz}-50 \mathrm{~Hz}]$ is considered and the results are given in Figure 11(b). It should be noticed that the first principal component represents mode 2 of the structure, as shown in Figure 10(b). Mode 2 which is more dominant than mode 1 is also more sensitive to damage. If only mode 1 is used, damages are only detected in cases $\# 2^{*}$ and $\# 3^{*}$ but they are detected in all cases $\# 0^{*}-\# 3^{*}$ when mode 2 is used. For the sake of conciseness, only the results for damage $\# 2^{*}$ are presented here as an example.

In the case of $\operatorname{PrC}$ slab, damages are detected much later and less apparent than in the RC slab, just before its collapse.

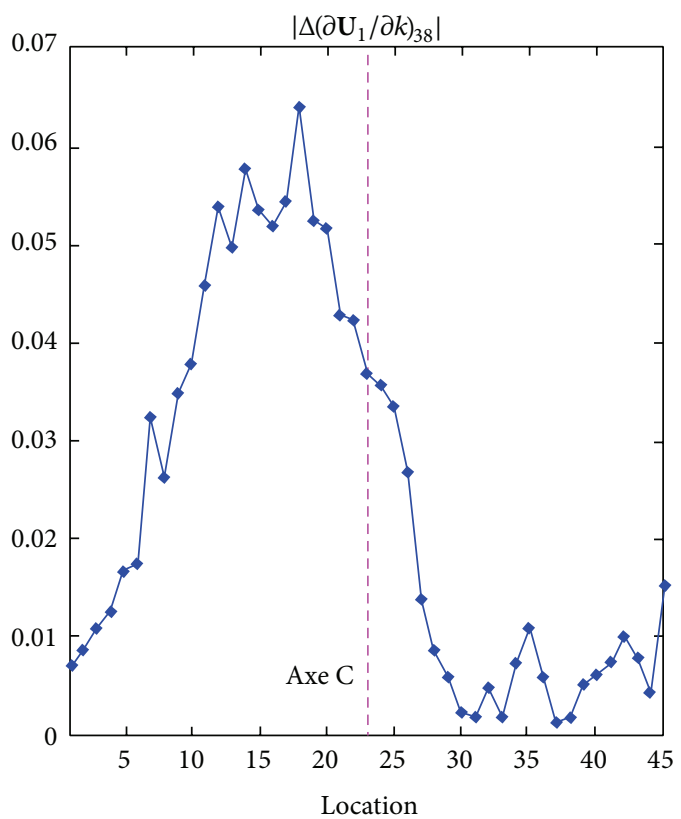

Figure 12: Damage localization in the PrC slab for damage \#2*, based on mode 2 .

It is confirmed by very small changes in frequencies under different conditions.

The localization procedure does not give any interesting outcome for the PrC slab when only mode 1 is considered. However, as in the RC slab, the use of mode 2 also allows a better localization. Damages $\# 3^{*}$ and $\# 2^{*}$ can be similarly localized as shown in Figure 12. The peak does not arise exactly at point 23 (along axis $\mathrm{C}$ ) but in the neighboring area. 


\section{Conclusion}

Several variants of Principal Component Analysis have been used in this study for detection and localization of damage. The advantage of PCA over classical modal identification methods relies on its easiness of use. The first results obtained on the Champangshiehl bridge are very encouraging. Furthermore, damage localization and the influence of environmental conditions on the diagnosis will be considered. The examples of the precast panels showed that the damages were better distinguished on the basis of the first eigenfrequency (especially for the RC slab) while they were localized in a more effective manner using the second mode-shape.

\section{Conflict of Interests}

The authors declare that there is no conflict of interests regarding the publication of this paper.

\section{Acknowledgment}

The author V. H. Nguyen is supported by the National Research Fund, Luxembourg.

\section{References}

[1] D. Montalvão, N. M. M. Maia, and A. M. R. Ribeiro, "A review of vibration-based structural health monitoring with special emphasis on composite materials," Shock and Vibration Digest, vol. 38, no. 4, pp. 295-324, 2006.

[2] W. Fan and P. Qiao, "Vibration-based damage identification methods: a review and comparative study," Structural Health Monitoring, vol. 10, no. 1, pp. 83-111, 2011.

[3] A.-M. Yan and J.-C. Golinval, "Null subspace-based damage detection of structures using vibration measurements," Mechanical Systems and Signal Processing, vol. 20, no. 3, pp. 611-626, 2006.

[4] V. H. Nguyen, Damage detection and fault diagnosis in mechanical systems using vibration signals [Ph.D. dissertation], University of Liège, 2010.

[5] V. H. Nguyen, C. Rutten, and J.-C. Golinval, "Fault diagnosis in industrial systems based on blind source separation techniques using one single vibration sensor," Shock and Vibration, vol. 19, no. 5, pp. 795-801, 2012.

[6] G. Kerschen and J.-C. Golinval, "Non-linear generalization of principal component analysis: from a global to a local approach," Journal of Sound and Vibration, vol. 254, no. 5, pp. 867-876, 2002.

[7] P. de Boe and J.-C. Golinval, "Principal component analysis of a piezosensor array for damage localization," Structural Health Monitoring, vol. 2, no. 2, pp. 137-144, 2003.

[8] P. V. Overschee and B. de Moor, Subspace Identification for Linear Systems: Theory, Implementation, Applications, Kluwer Academic Publishers, Dordrecht, The Netherlands, 1997.

[9] G. H. Golub and C. F. van Loan, Matrix Computations, The Johns Hopkins University Press, Baltimore, Md, USA, 1996.

[10] J. Mahowald, S. Maas, D. Waldmann, A. Zürbes, and F. Scherbaum, "Damage identification and localisation using changes in modal parameters for civil engineering structures," in Proceedings of the International Conference on Noise and Vibration Engineering, pp. 1103-1117, Leuven, Belgium, 2012.

[11] B. Peeters and G. de Roeck, "Stochastic system identification for operational modal analysis: a review," Journal of Dynamic Systems, Measurement and Control, vol. 123, no. 4, pp. 659-667, 2001.

[12] J. Mahowald, S. Maas, F. Scherbaum, D. Waldmann, and A. Zürbes, "Dynamic damage identification using linear and nonlinear testing methods on a two-span prestressed concrete bridge," in Proceedings of the 3rd International Symposium on Life-Cycle Civil Engineering (IALCCE '12), pp. 157-164, CRC Press, Vienna, Austria, October 2012.

[13] J. Mahowald, V. Bungard, D. Waldmann, S. Maas, A. Zürbes, and G. de Roeck, "Comparison of linear and nonlinear static and dynamic behaviour of prestressed and non-prestressed concrete slab elements," in Proceedings of the International Conference on Noise and Vibration Engineering, pp. 717-728, Leuven, Belgium, 2010.

[14] J. V. Araújo dos Santos, N. M. M. Maia, C. M. Mota Soares, and C. A. Mota Soares, "Structural damage identification: a survey," in Trends in Computational Structures Technology, B. H. V. Topping and M. Papadrakakis, Eds., chapter 1, pp. 1-24, SaxeCoburg Publications, Stirlingshire, UK, 2008.

[15] L. J. Jiang and K. W. Wang, "An experiment-based frequency sensitivity enhancing control approach for structural damage detection," Smart Materials and Structures, vol. 18, no. 6, Article ID 065005, 2009.

[16] V.H. Nguyen and J.-C. Golinval, "Damage localization in linearform structures based on sensitivity investigation for principal component analysis," Journal of Sound and Vibration, vol. 329, no. 21, pp. 4550-4566, 2010.

[17] D. Todd Griffith, "Analytical sensitivities for principal components analysis of dynamical systems," in Proceedings of the 27th IMAC Conference \& Exposition on Structural Dynamics, Orlando, Fla, USA, February 2009.

[18] J. L. Junkins and Y. Kim, Introduction to Dynamics and Control of Flexible Structures, AIAA Education Series, American Institute of Aeronautics and Astronautics, Reston, Va, USA, 1993. 

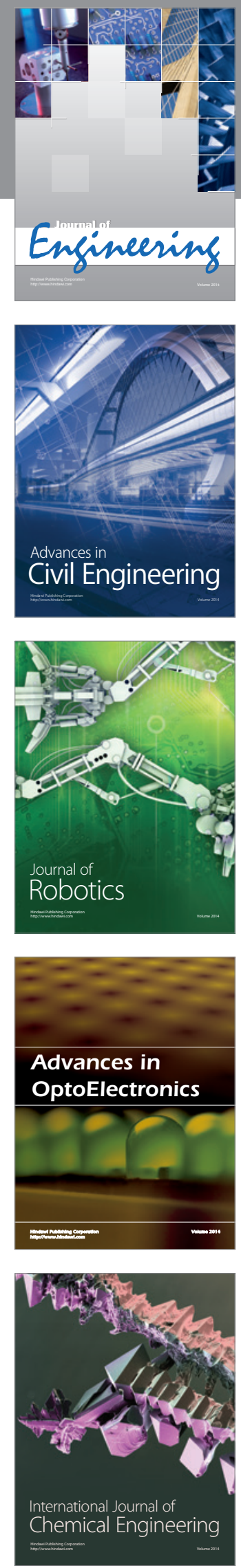

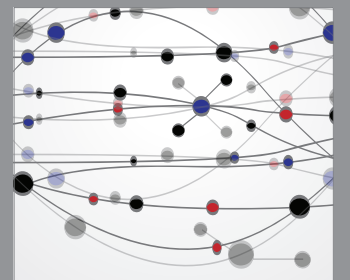

The Scientific World Journal
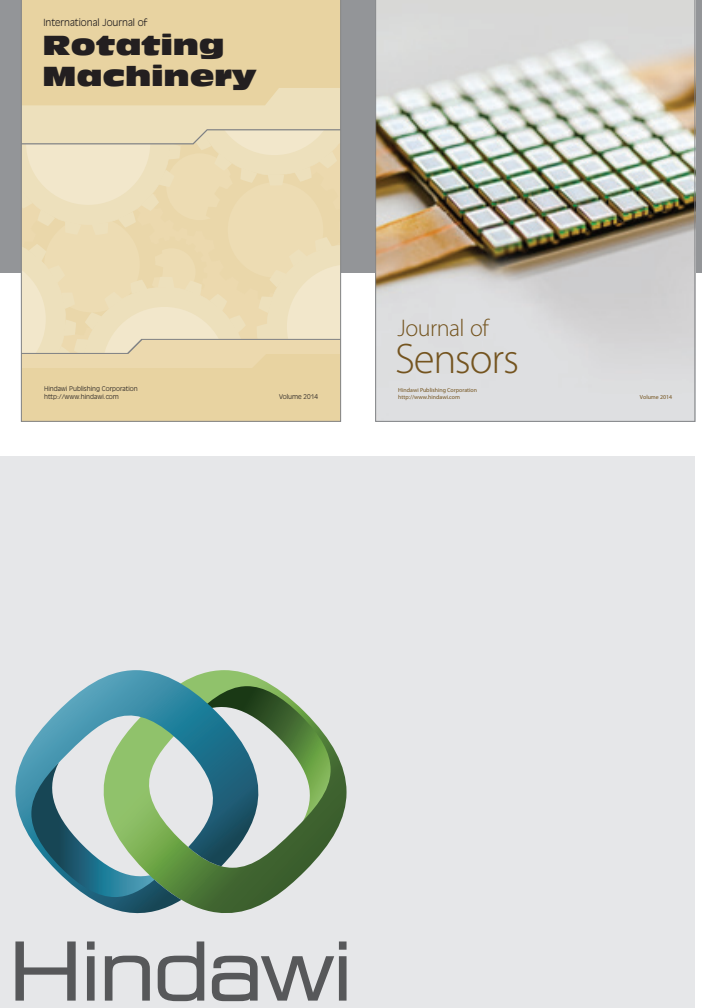

Submit your manuscripts at http://www.hindawi.com
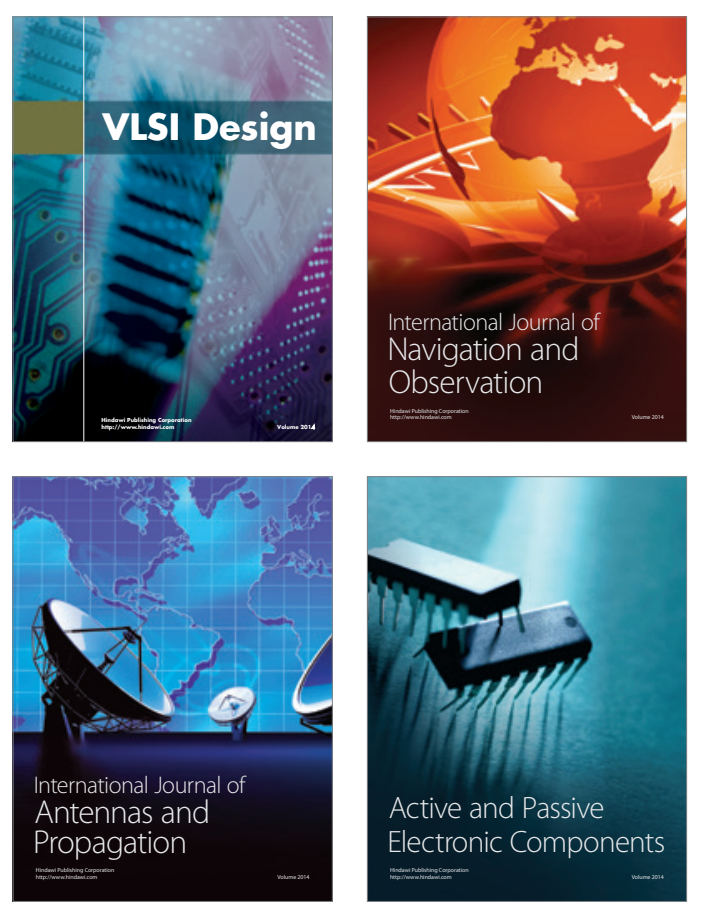
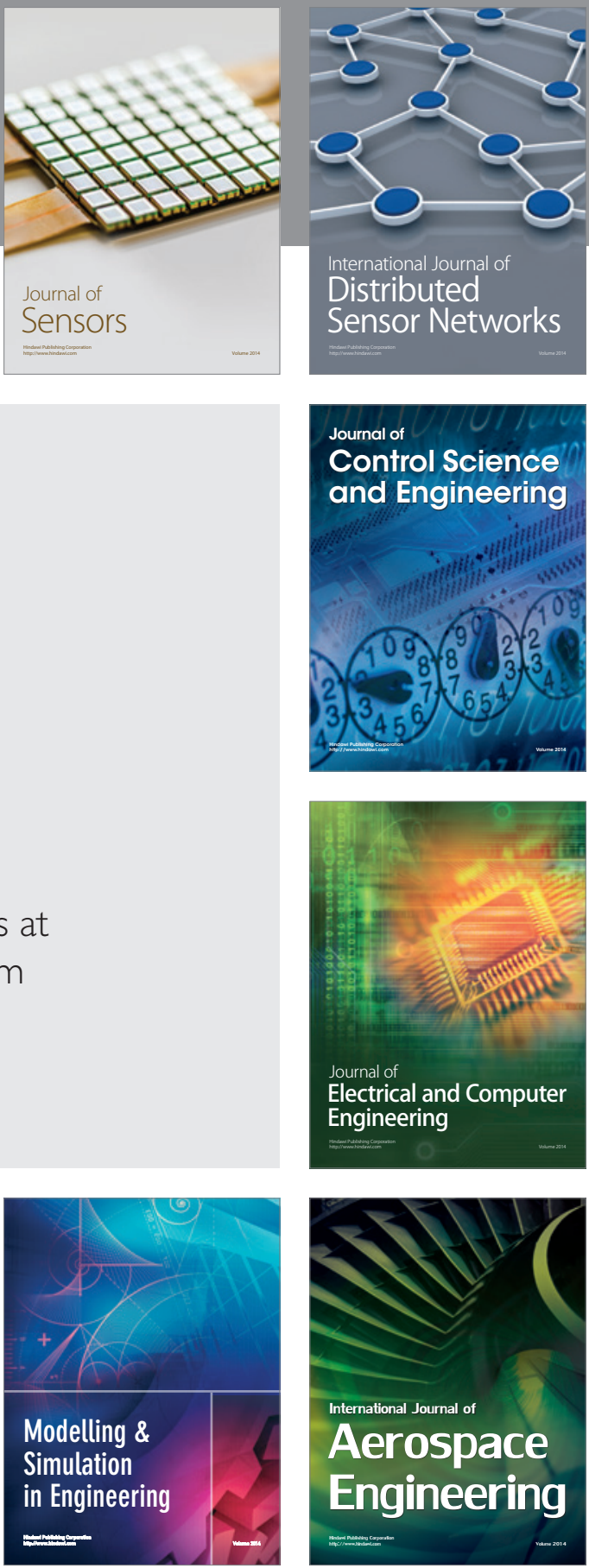

Journal of

Control Science

and Engineering
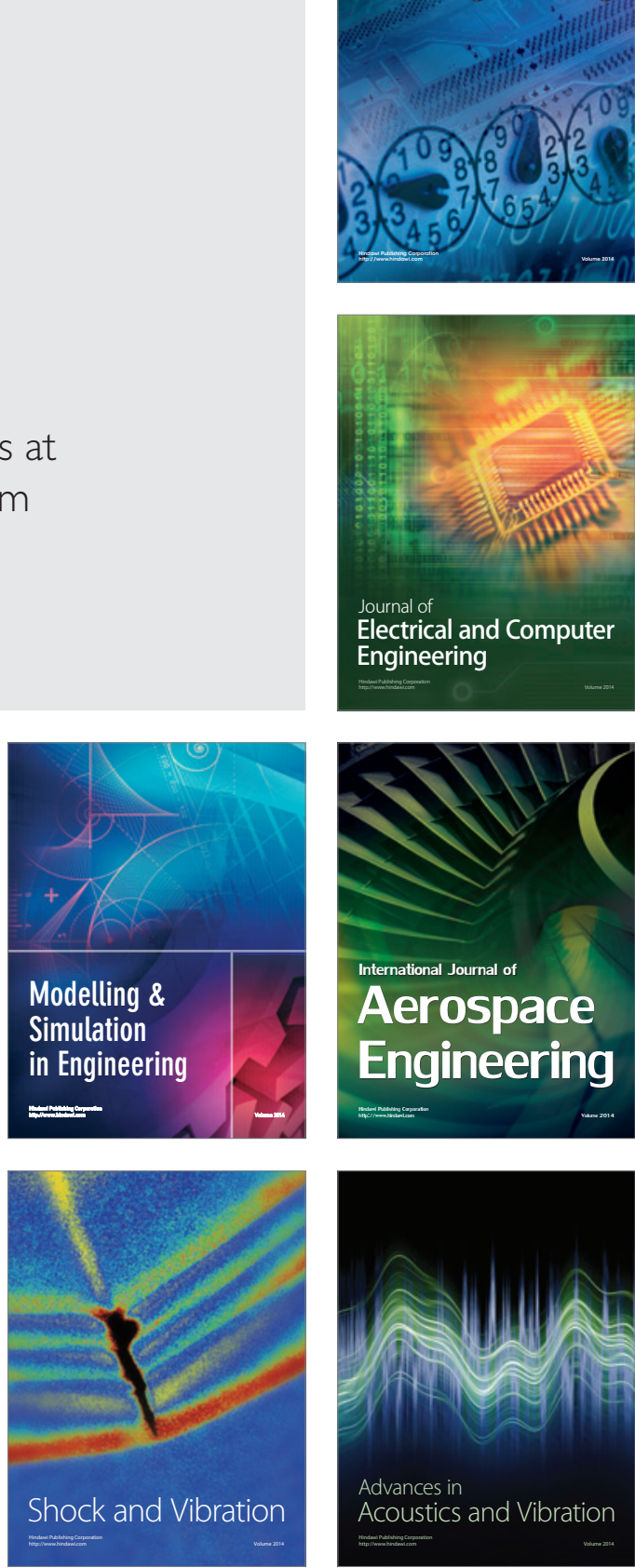